\title{
Covalent Organic Framework Films through Electrophoretic Deposition - Creating Efficient Morphologies for Catalysis
}

Julian M. Rotter, a Simon Weinberger, ${ }^{a}$ Jonathan Kampmann, ${ }^{\text {a }}$ Torben Sick, ${ }^{\text {a }}$ Menny Shalom, ${ }^{\mathrm{b}}$ Thomas Bein ${ }^{\mathrm{a}}$ and Dana D. Medina, ${ }^{\mathrm{a}, *}$

a Department of Chemistry and Center for NanoScience (CeNS), University of Munich (LMU), Butenandtstr. 5-13, 81377 Munich, Germany

b. Department of Chemistry and Ilse Katz Institute for Nanoscale Science and Technology, Ben-Gurion University of the Negev Beer-Sheva 8410501, Israel

\section{Table of Contents}

Deposition of BDT-ETTA COF on FTO, ITO and titanium foil ...................................................

Deposition of BDT-ETTA COF in anisole, ethyl acetate and toluene...............................................

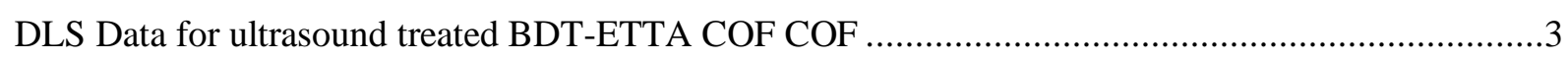

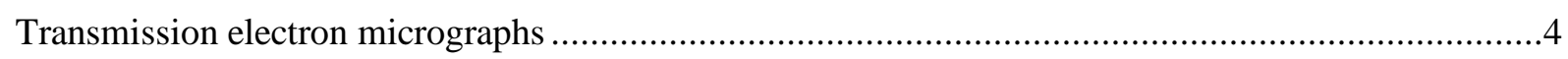

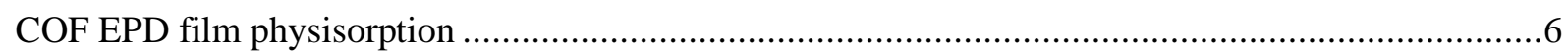

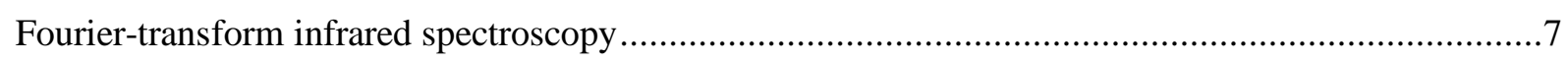

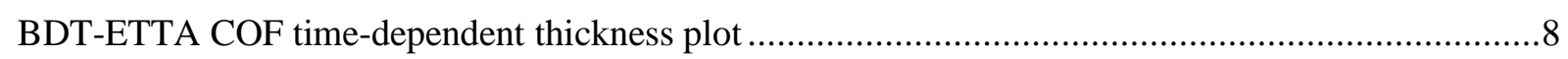

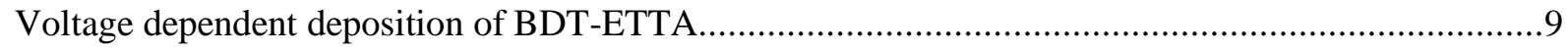

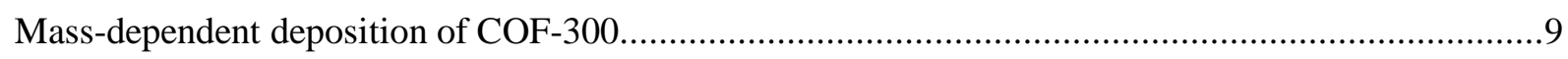

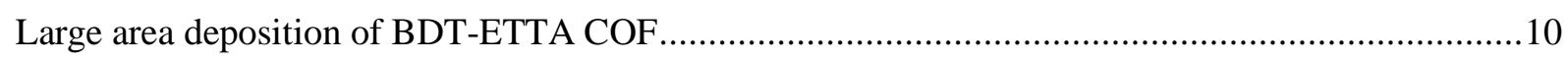

SEM Micrographs of BDT-ETTA COF deposited on a porous mesh ............................................10

Thickness dependent PEC current measurements and chronoamperometry ......................................11

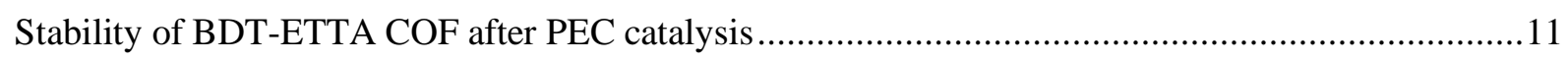

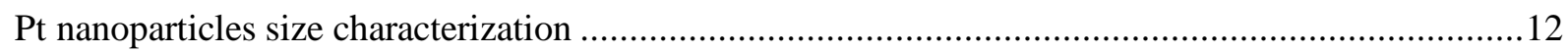




\section{Deposition of BDT-ETTA COF on FTO, ITO and titanium foil}

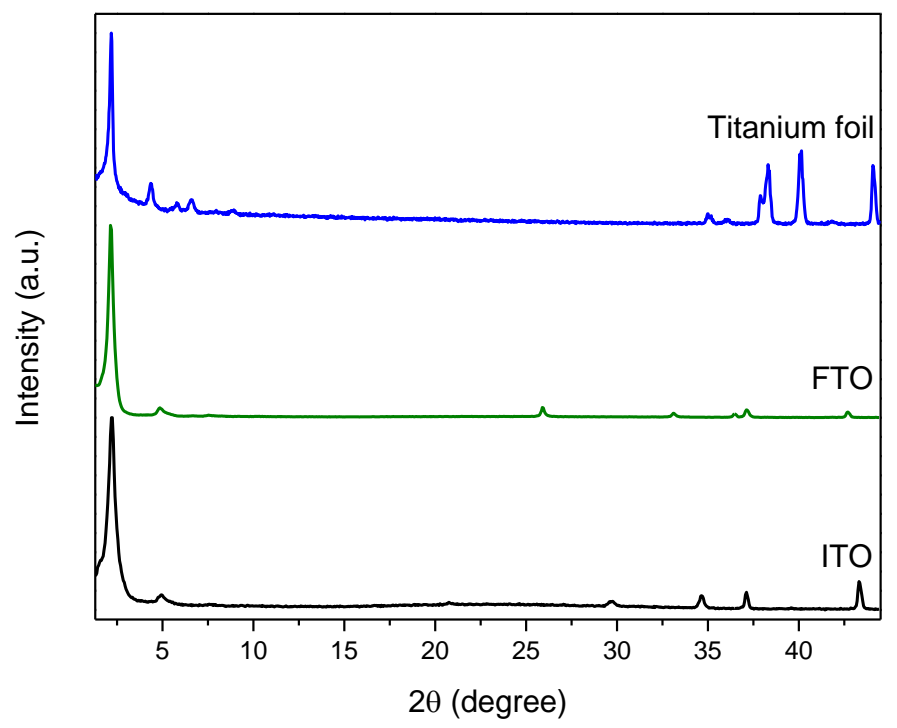

Figure S1: Deposition of BDT-ETTA COF on different conducting surfaces, namely titanium foil, glass coated with FTO and glass coated with ITO. All depositions were carried out using $10 \mathrm{~mL}$ of BDT-ETTA COF suspension in ethyl acetate at 900 $\mathrm{V}$ for $2 \mathrm{~min}$.

\section{Deposition of BDT-ETTA COF in anisole, ethyl acetate and toluene}

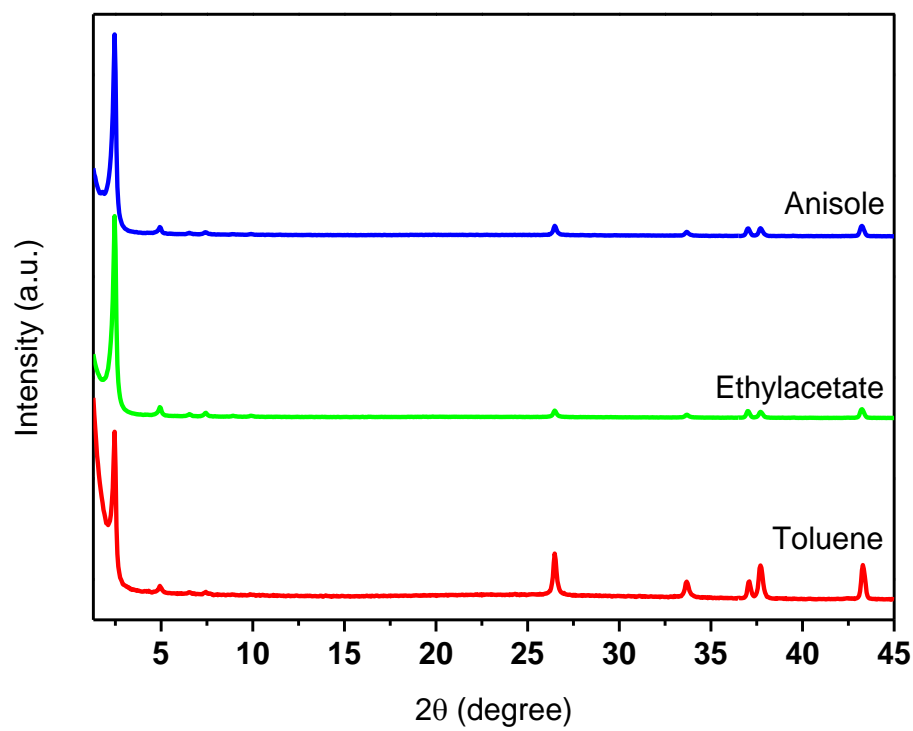

Figure S2: Deposition of BDT-ETTA COF from different solvents, namely anisole, ethyl acetate and toluene. All depositions were carried out using $10 \mathrm{~mL}$ of the respective BDT-ETTA COF suspension at $900 \mathrm{~V}$ for $2 \mathrm{~min}$. 
DLS Data for ultrasound treated BDT-ETTA COF

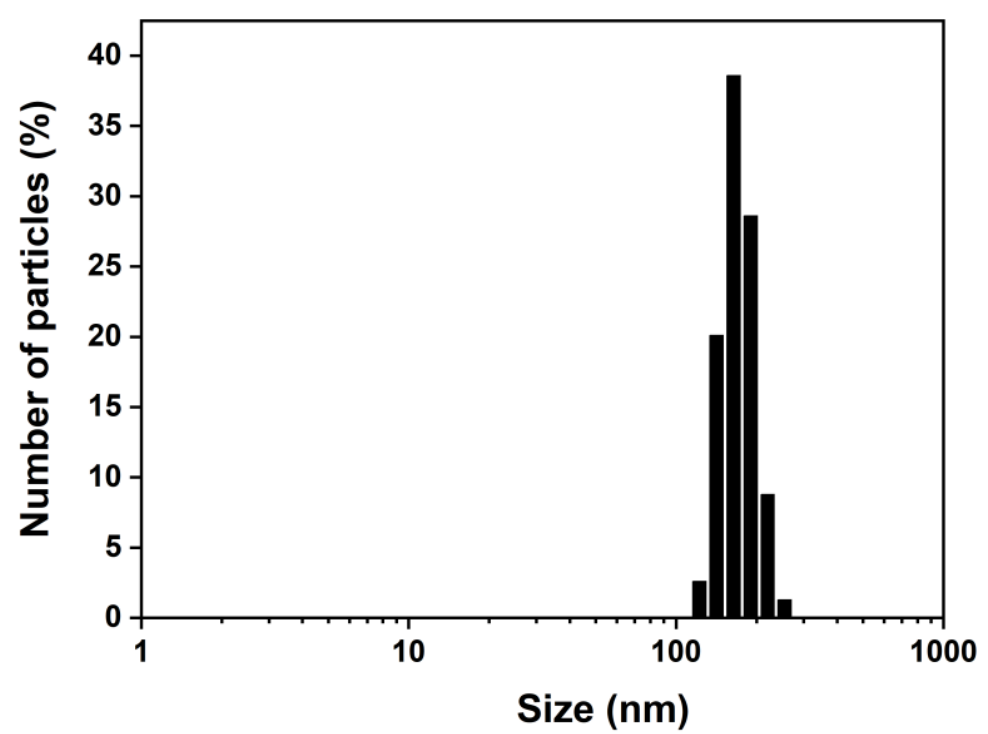

Figure S3: Particle size distribution of BDT-ETTA COF after the ultrasonic milling process. 


\section{Transmission electron micrographs}

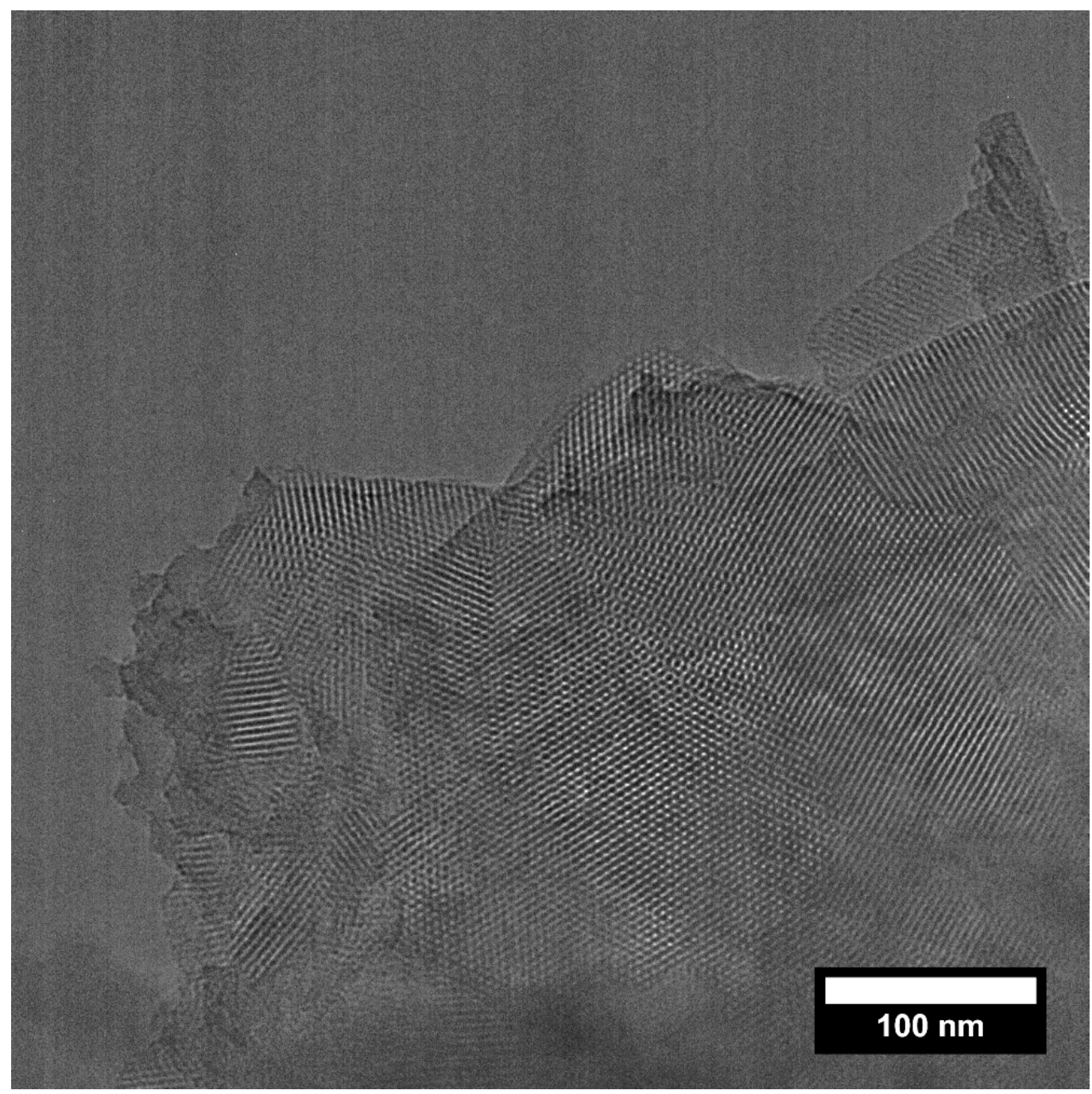

Figure S4: TEM image of deposited BDT-ETTA COF particles after the ultrasonic milling process. 


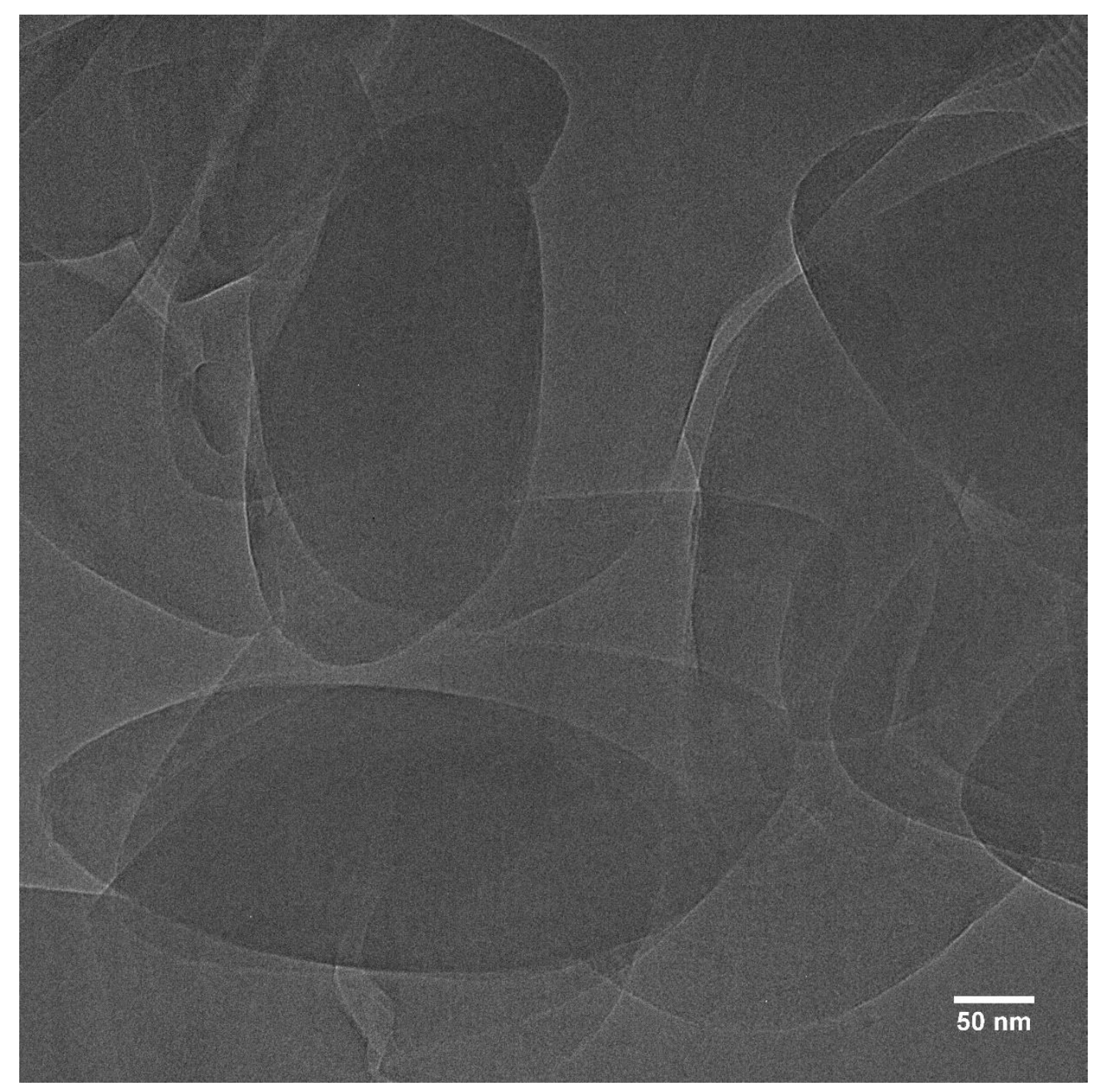

Figure S5: TEM image of deposited COF-300 particles. 


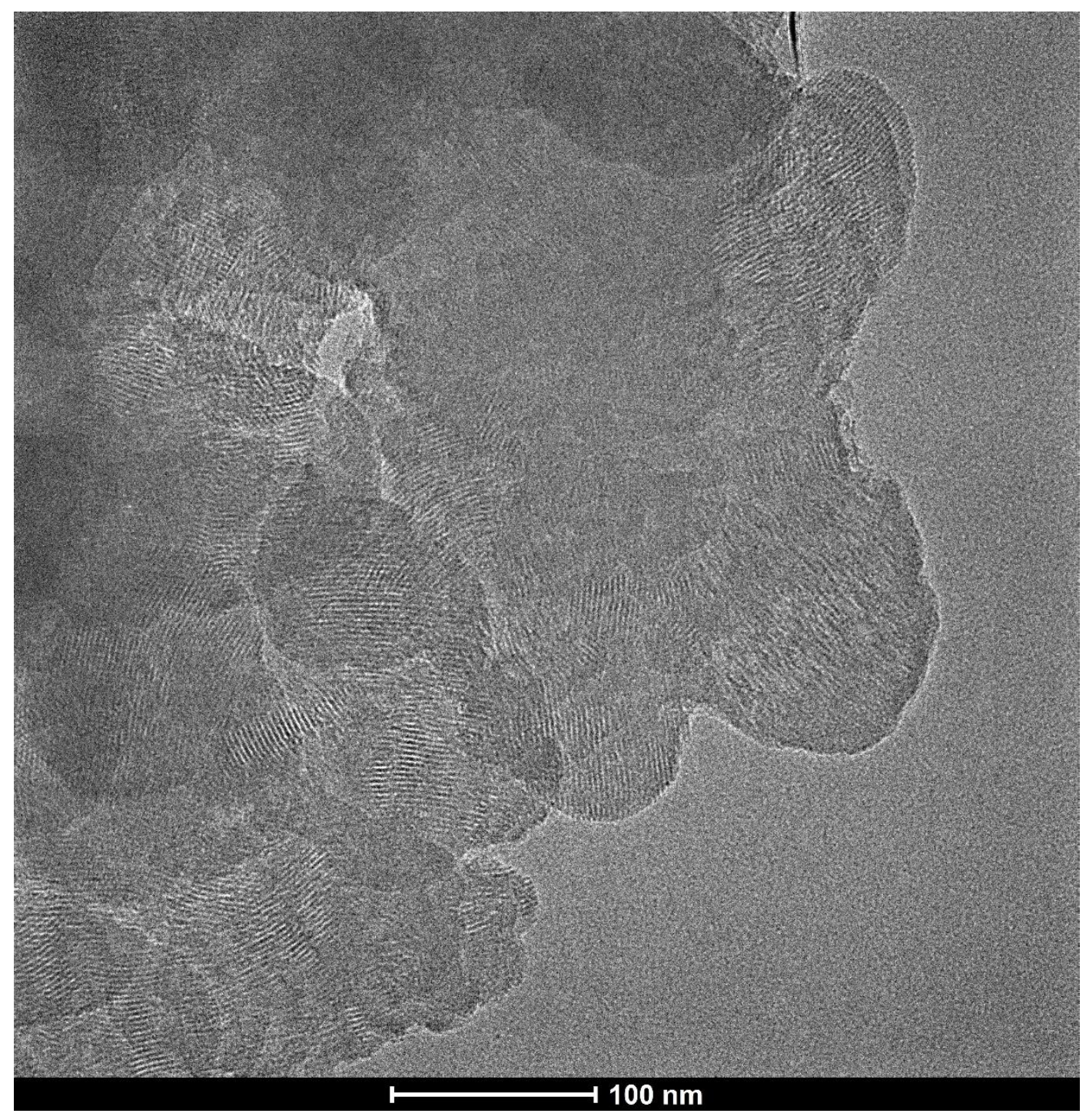

Figure S6: TEM image of deposited COF-5.

\section{COF EPD film physisorption}

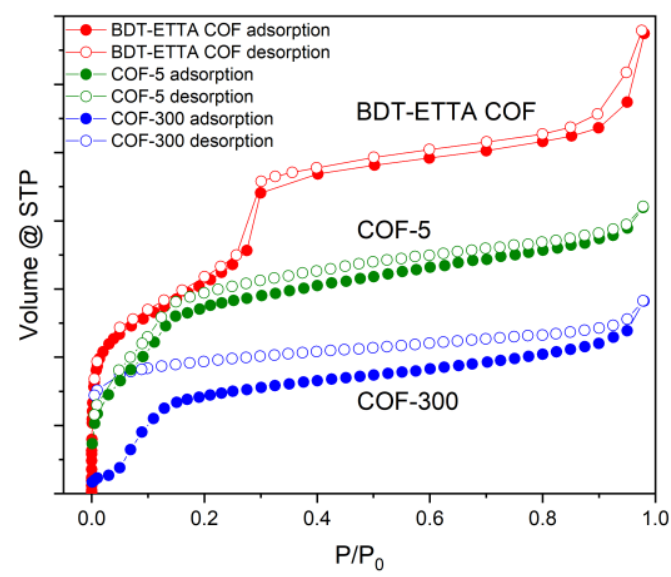

Figure S7: Nitrogen physisorption isotherms of scratched-off powder from the respective COF EPD films. Due to the low mass of the scratched-off powders, quantitative nitrogen uptake could not be accurately determined. 


\section{Fourier-transform infrared spectroscopy}
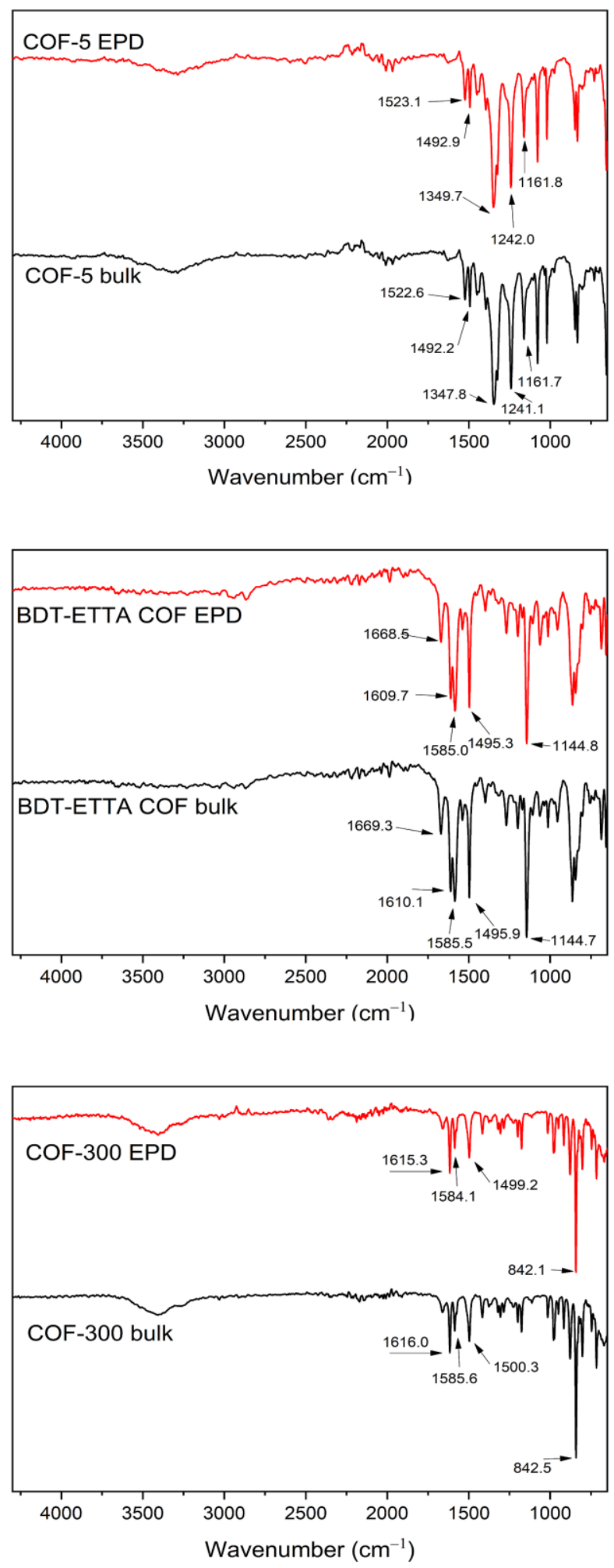

Figure S8: FT-IR spectra of the as-synthesized bulk material and as scratched-off EPD film materials. In all three cases IR vibrations are preserved. This indicates that no chemical degradation occurred during the EPD process. 
BDT-ETTA COF time-dependent thickness plot

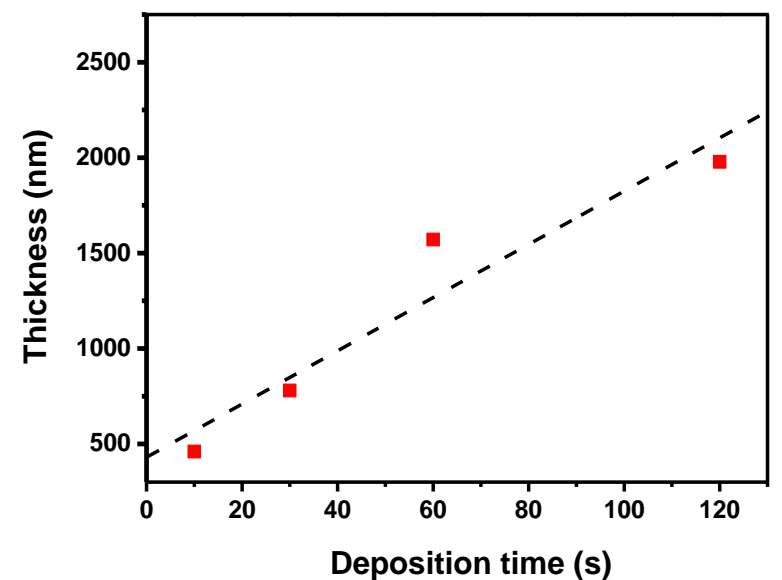

Figure S9: Time-dependent thickness plot of BDT-ETTA COF deposited from ethyl acetate at $900 \mathrm{~V}$. Thicknesses obtained from SEM cross-sections. 


\section{Voltage dependent deposition of BDT-ETTA COF}

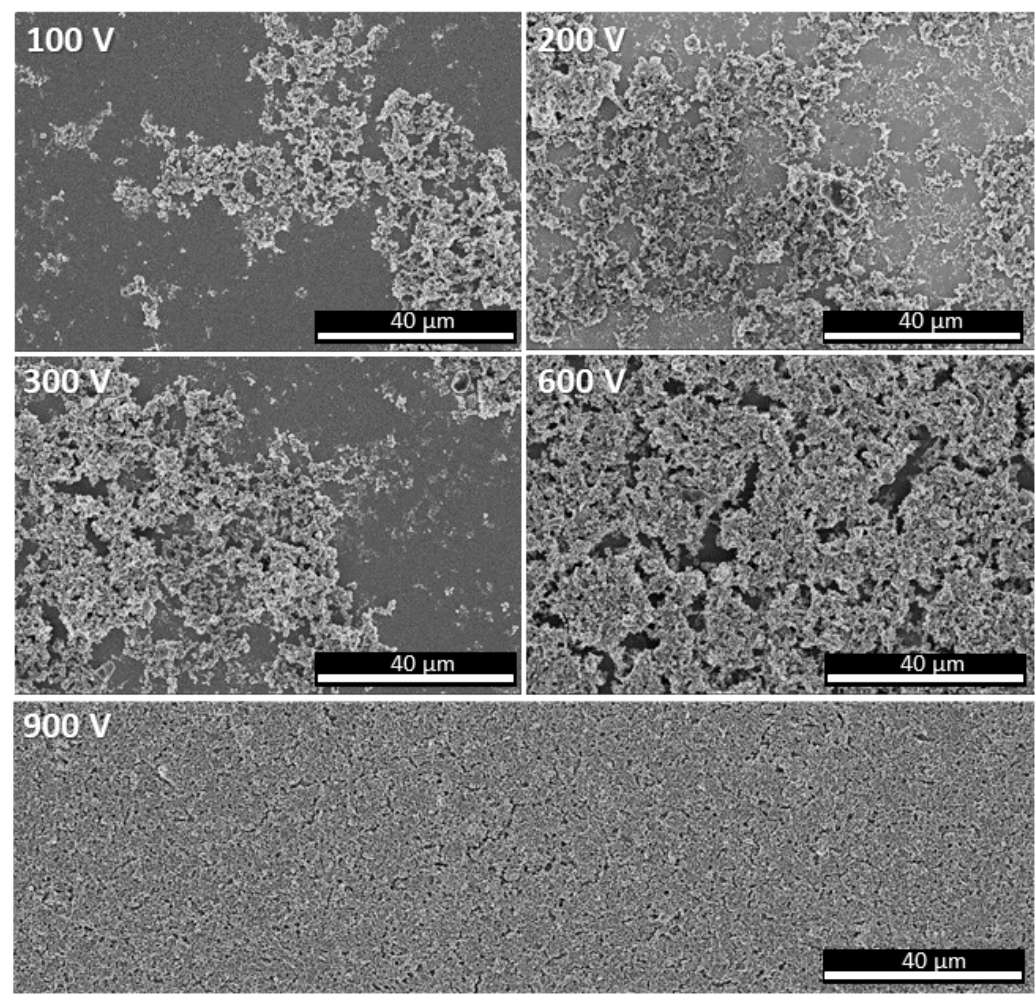

Figure S10: SEM top-view images of BDT-ETTA COF deposited at different voltages for 2 min from ethyl acetate.

\section{Mass-dependent deposition of COF-300}
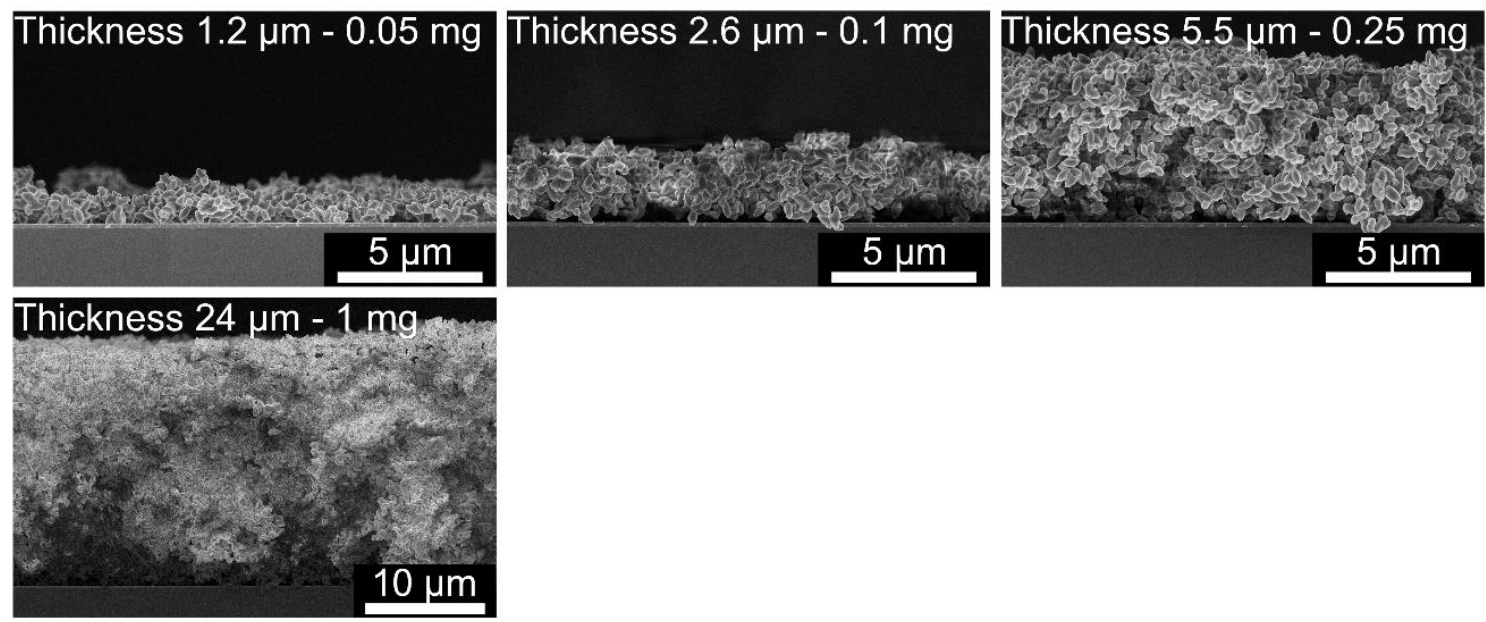

Figure S11: SEM cross-section images of depositions of COF-300 using different masses in $10 \mathrm{~mL}$ suspension and the resulting film thicknesses. Depositions were carried out at $900 \mathrm{~V}$ for $2 \mathrm{~min}$ from ethyl acetate. 


\section{Large area deposition of BDT-ETTA COF}

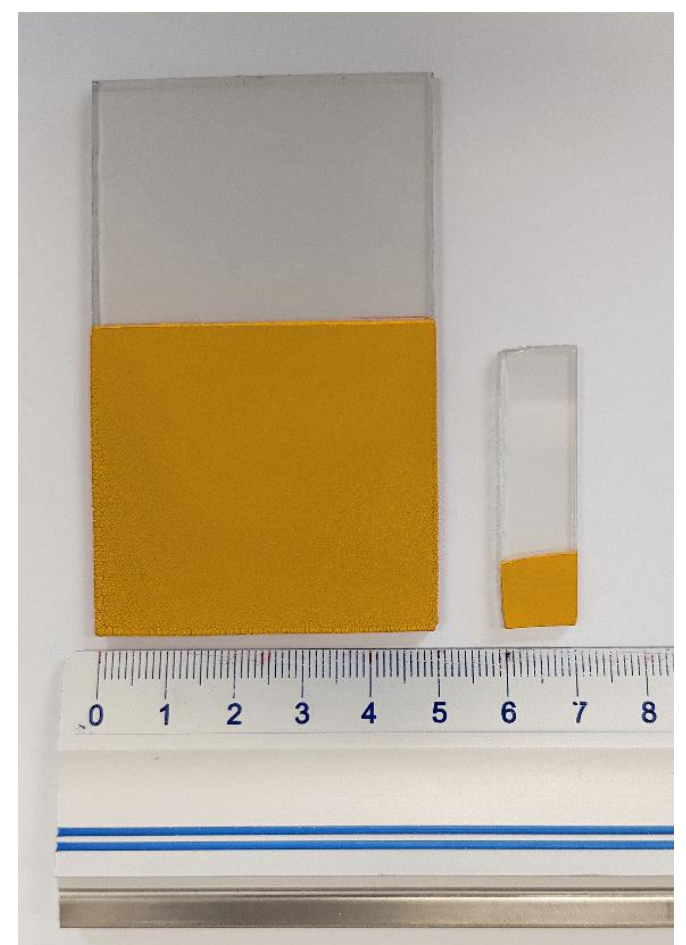

Figure S12: Photograph of an EPD of BDT-ETTA COF on $5 \mathrm{~cm} \times 5 \mathrm{~cm}$ electrode area as well as a $1 \mathrm{~cm} \times 1 \mathrm{~cm}$ film. Both depositions were carried out on FTO at $900 \mathrm{~V}$ for 2 min.

\section{SEM Micrographs of BDT-ETTA COF deposited on a porous mesh}
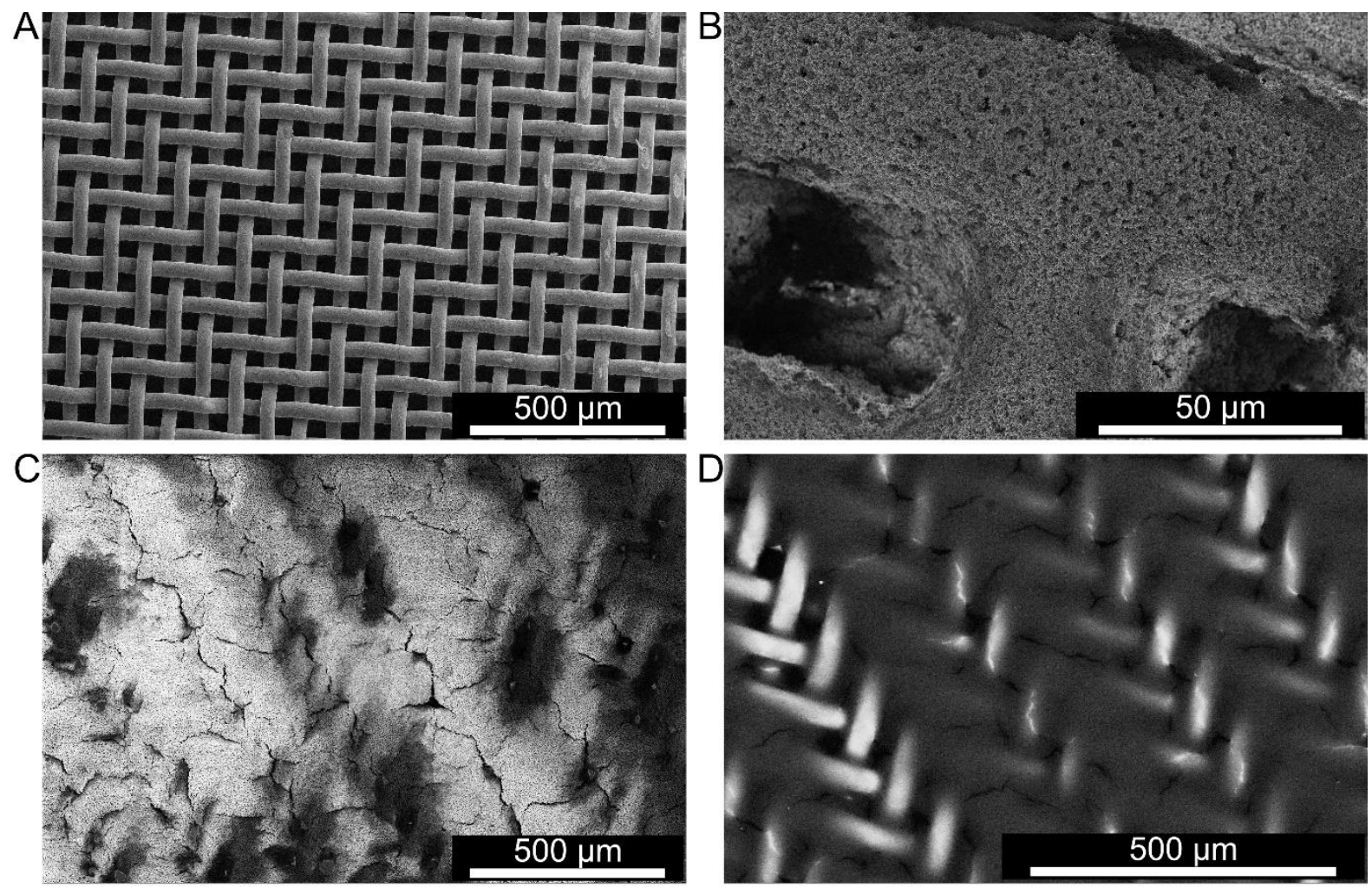

Figure S13: SEM top view micrographs of BDT-ETTA COF depositions on a porous steel mesh (mesh size 270). (A) Image of the bare mesh prior to deposition. (B) High magnification of BDT-ETTA COF particles deposited on the mesh. (C) Deposition of BDT-ETTA COF in high concentration on a steel mesh, revealing the complete coverage of the pores. (D) Corresponding back-scattered electron micrograph at $30 \mathrm{kV}$ acceleration voltage, revealing the underlying mesh structure. 


\section{Thickness dependent PEC current measurements and chronoamperometry}
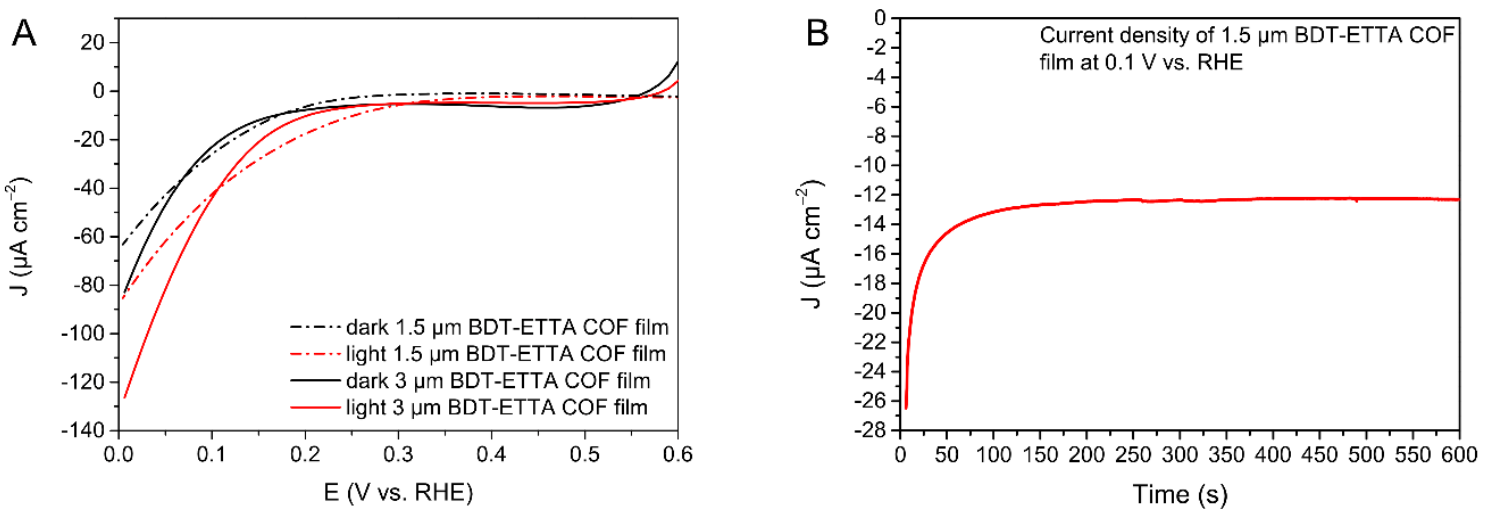

Figure S14: (A) Thickness dependent PEC linear sweep voltammograms of electrodes coated with BDT-ETTA COF. Illumination at AM1.5G. (B) Chronoamperometric current density measurement of BDT-ETTA COF under illumination.

\section{Stability of BDT-ETTA COF after PEC catalysis}

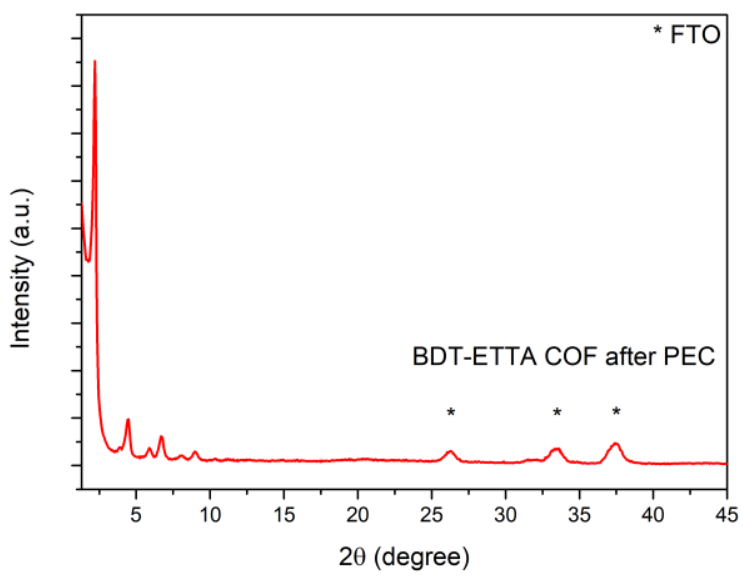

Figure S15: Stability of BDT-ETTA COF after 30 minutes of chopped illumination at $0.1 \mathrm{~V}$ vs. RHE. 


\section{Pt nanoparticles size characterization}

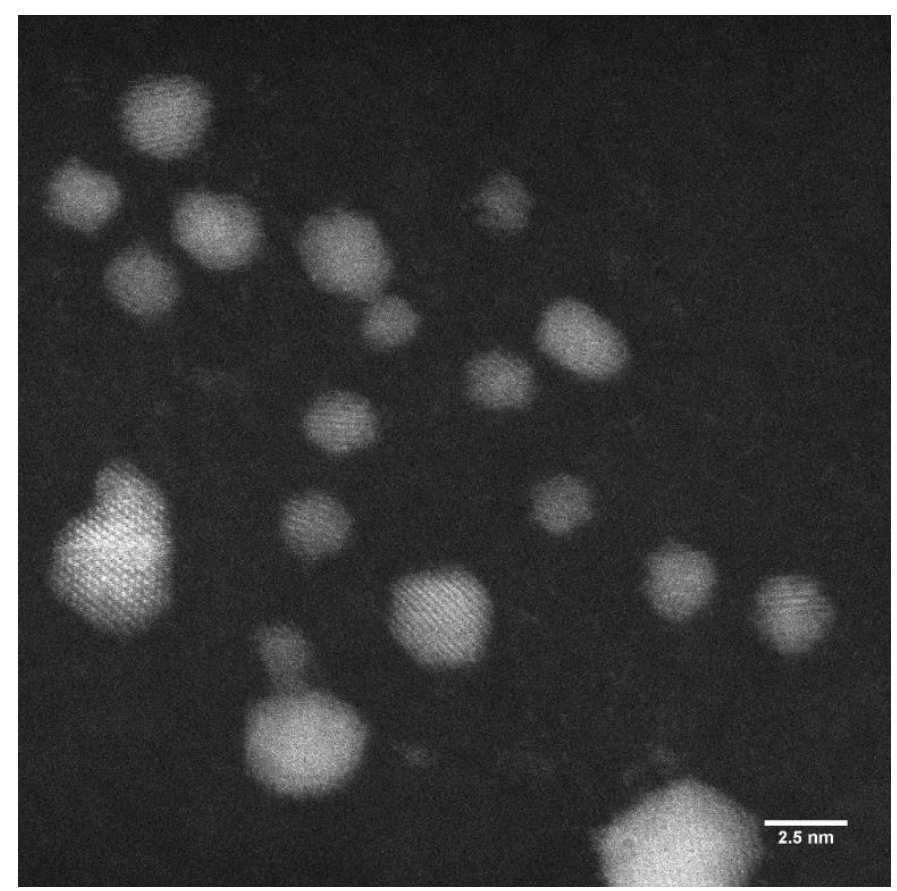

Figure S16: TEM micrograph of Pt nanoparticles after lyophilization.

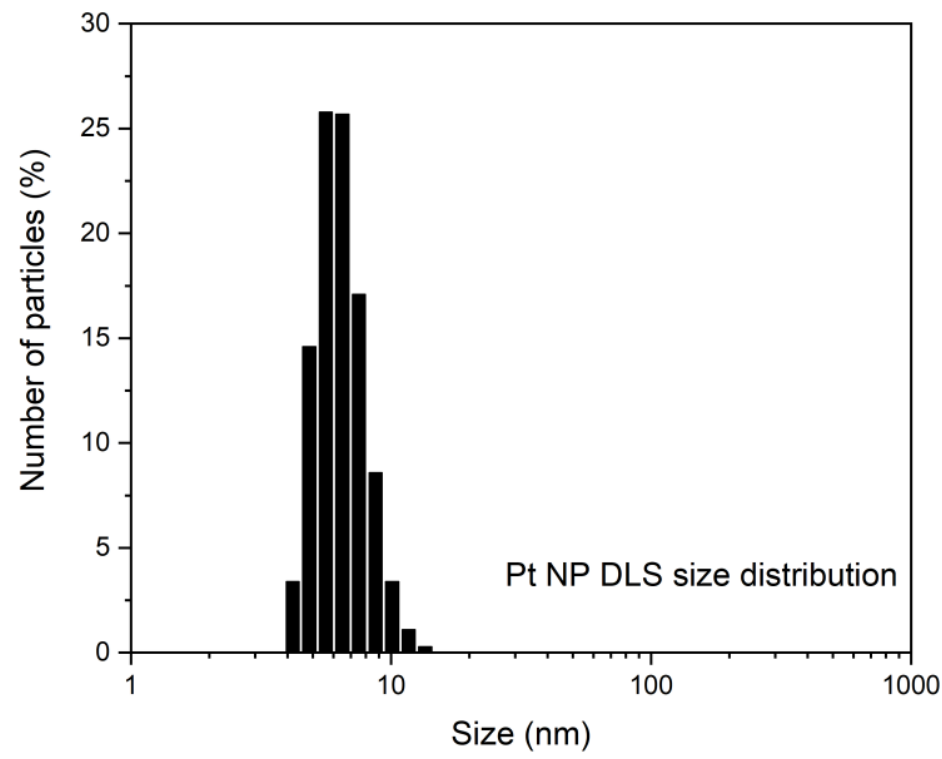

Figure S17: DLS profile of Pt nanoparticles after lyophilization. 